\title{
Convergent Dirichlet series
}

\author{
By Henry Helson
}

\section{Introduction}

This note presents several results about Dirichlet series

$$
f(s)=\sum_{n=1}^{\infty} a_{n} e^{-\lambda n s} \quad(s=\sigma+i t),
$$

which are loosely connected by a common idea of proof, rather than by any formal dependence. Section 2 contains an elementary lemma, really a reformulation of the well-known formula expressing the abscissa of convergence of (1) in terms of the coefficients and exponents of the series. After this lemma, the sections can be read independently.

Section 3 treats the convergence problem for Dirichlet series: to determine the abscissa of convergence from properties of the function $f$. A famous and difficult theorem of Landau and Schnee gives sufficient conditions for (1) to converge in a region $\sigma>\sigma_{0}$. A restriction has to be put on the exponents $\lambda_{n}$ as well as on $f$. Without any restriction on the exponents beyond the fundamental one

$$
\lambda_{1}<\lambda_{2}<\ldots ; \quad \lambda_{n} \rightarrow \infty,
$$

we shall show (within the half-plane $\sigma>0$ ) that the series converges precisely as far to the left as $f(\sigma+i t) /(\sigma+i t)$ satisfies a uniform condition of growth, and as a function of $t$ is the Fourier transform of a function which is summable and boun. ded. This theorem is superficial, and it does not lead easily to new convergence theorems. In particular, the new theorem does not obviously imply the theorem of Landau and Schnee. But the result shows why convergence theorems are difficult to prove: they amount to showing that functions with given properties are Fourier transforms, and simple criteria on which to decide do not exist.

In Section 4 we reconsider the formula of Perron:

$$
\sum_{\lambda_{n} \leqslant x}^{\prime} a_{n}=\lim _{\omega \rightarrow \infty} \frac{1}{2 \pi i} \int_{\sigma-i \omega}^{\sigma+i \omega} \frac{f(s)}{s} e^{i x s} d s .
$$

The stroke on the sign of summation means, here and hereafter, that the final term is to be halved if the last $\lambda_{n}$ is $x$. The formula holds for $\sigma>\sigma_{1}$, where (for the rest of the paper) $\sigma_{1}$ denotes the abscissa of convergence $\sigma_{c}$ if that is positive, and 0 otherwise. 


\section{H. HELSON, Convergent Dirichlet series}

Ordinarily (3) is proved by means of Cauchy's Theorem. The content of this section is that the formula, slightly rewritten, is at first glance an instance of the simplest Fourier inversion formula. The inversion formula is not very easy to prove from first principles, so this proof of $(3)$ is not in the end particularly simple, but it is surely time to rescue this branch of Analysis from total and unnecessary dependence on the methods of complex function theory.

That goal is pursued a little further in Section 6 , where we give a simple proof of this theorem of Schnee: for $\sigma>\sigma_{c}$,

$$
\lim _{\omega \rightarrow \infty} \frac{1}{2 \omega} \int_{-\omega}^{\omega} f(\sigma+i t) e^{i \lambda t} d t=a_{n} e^{-\lambda_{n} \sigma} \text { or } \quad 0
$$

depending on whether $\lambda=\lambda_{n}$, or $\lambda$ is no $\lambda_{n}$.

The theorem is usually given in two forms: the relation (4) holds in the region of uniform convergence first; and then in the region of convergence under a supplementary hypothesis on the exponents. Carlson intimates [2, p. 10] that he knows the full result, but his remarks there do not substantiate the fact, and apparently no proof has been published. At any rate the proof given here is simple, and it shows that this convergence theorem (like Perron's Theorem and the convergence theorems for trigonometric series) rests finally on the RiemannLebesgue Lemma.

Section 5 contains the main new result of the paper. Perron's Theorem suggests that the discrete sum analogous to the right side of (3)

$$
\sum_{-N}^{N} \frac{f(\sigma+i k)}{\sigma+i k} e^{i k x}
$$

might have a limit as $N$ tends to infinity. Extended formally to infinity, (5) is the Fourier series of a function easily written down which has limits from left and right at each point, but which generally is not of bounded variation. The convergence of (5) does not seem to follow from known theorems about the convergence of Fourier series. We establish convergence in the region $\sigma>\sigma_{1}$, under a mild hypothesis bearing on the exponents.

Only the most elementary results about Dirichlet series have been used in the paper, but the references $[1,3,5]$ will orient the reader who, like the author, is a newcomer to the subject. In particular, the theorems treated here are related to Landau's treatise [3] at pages $828,782,905$, and 945 .

\section{2.}

To the series (1) we associate functions

$$
F(x)=\sum_{\lambda_{n} \leqslant x}^{\prime} a_{n}, \quad F_{\sigma}(x)=\sum_{\lambda_{n} \leqslant x}^{\prime} a_{n} e^{-\lambda_{n} \sigma}
$$

Each one vanishes for $x<\lambda_{1}$. Evidently (1) converges for $s=\sigma$ if and only if $F_{\sigma}(x)$ has a limit as $x$ tends to infinity.

These functions are related by the formulas 


$$
\left.\begin{array}{l}
F_{\sigma}(x)=e^{-x \sigma} F(x)+\sigma \int_{-\infty}^{x} e^{-u \sigma} F(u) d u \\
F(x)=e^{x \sigma} F_{\sigma}(x)-\sigma \int_{-\infty}^{x} e^{u \sigma} F_{\sigma}(u) d u
\end{array}\right\}
$$

which are verified by means of an integration by parts on the right side. At points of discontinuity (namely the points $\lambda_{n}$ ) we have to add that $F$ and $F_{\sigma}$ have been defined to be averages of their left-hand and right-hand limits.

Lemma 1. For $\sigma>0$

(a) If $F_{\sigma}(x)$ converges as $x$ tends to infinity, then $e^{-x \sigma} F^{\prime}(x)$ tends to zero;

(b) If $F(x)=O\left(e^{x \sigma}\right)$, then $F_{\sigma}(x)=O(x)$ and $F_{\sigma^{\prime}}(x)$ converges for each $\sigma^{\prime}>\sigma$; and consequently

(c) If $\alpha$ is the infimum of numbers $\beta$ such that $F(x)=O\left(e^{x \beta}\right)$, then $\sigma_{c}=\alpha$ provided either number is positive.

These facts are easy to derive from (7).

\section{The convergence problem}

In this section it will be convenient to assume that $\lambda_{1} \geqslant 0$. We have defined $\sigma_{1}$ to be the maximum of $\sigma_{c}$ and 0 . By Lemma $1, e^{-x \sigma} F(x)$ is summable and tends to zero if $\sigma>\sigma_{1}$. Using this fact, an integration by parts shows that

$$
\int_{-\infty}^{\infty} F(x) e^{-x s} d x=\frac{f(s)}{s} \quad\left(\sigma>\sigma_{1}\right)
$$

so that $f(s) / s$, as a function of $t$, is the Fourier transform of a function which is bounded and summable, provided $\sigma>\sigma_{1}$.

If $e^{-x \sigma} F(x)$ is bounded and summable, then it is square-summable as well, so that

$$
\int_{-\infty}^{\infty}\left|F(x) e^{-x \sigma}\right|^{2} d x=\frac{1}{2 \pi} \int_{-\infty}^{\infty}\left|\frac{f(\sigma+i t)}{\sigma+i t}\right|^{2} d t<\infty
$$

for $\sigma>\sigma_{1}$. Moreover it is obvious that the left side of (9) decreases as $\sigma$ increases (using the assumption that $\lambda_{1} \geqslant 0$ ), so that the right side is uniformly smaller than a constant for $\sigma>\sigma_{1}+\varepsilon$ (each $\varepsilon>0$ ).

We wish to prove that these properties characterize $\sigma_{1}$, or, precisely,

Theorem 1. $\sigma_{1}$ is the infimum of positive numbers $\beta$ with these properties: the right side of (9) is finite and uniformly bounded for $\sigma>\beta$; and $f(\sigma+i t) /(\sigma+i t)$ is the Fourier transform of a function which is bounded and summable, for each $\sigma>\beta$.

We only have to prove that if $\beta$ is a positive number having the properties of the theorem, then $F(x)=\mathrm{O}\left(e^{x \sigma}\right)$ for each $\sigma>\beta$. For then, by Lemma 1, we have $\sigma_{1} \leqslant \beta$, and the inequality persists for the infimum of such $\beta$. The opposite inequality has already been established.

By hypothesis, there is for each $\sigma>\beta$ a function $G_{\sigma}$ which is summable and bounded, and for which 


$$
\frac{f(\sigma+i t)}{\sigma+i t}=\int_{-\infty}^{\infty} G_{\sigma}(x) e^{-i x t} d x
$$

The assumption that the right side of (9) is uniformly bounded for $\sigma>\beta$ implies, by a theorem of Paley and Wiener $\left[4\right.$, p. 8], that $G_{\sigma}$ has the special form $G(x) e^{-x \sigma}$, where $G$ is a single function vanishing for negative values of $x$. But for $\sigma>\sigma_{1}$ the representation (8) is valid as well as (10), and the unicity theorem for Fourier transforms imples that

$$
G(x) e^{-x \sigma}=F(x) e^{-x \sigma} \quad\left(\sigma>\sigma_{1}\right) .
$$

Hence $G=F$ almost everywhere and so $F(x) e^{-x \sigma}$ is bounded and summable for $\sigma>\beta$. This concludes the proof of the theorem.

Theorem 1 might be generalized in two directions. In the proof it was assumed that (1) possesses a region of convergence. It should be possible to prove this fact, as part of the theorem, on the weaker hypothesis that (1) is summable in some region.

Secondly, the same method ought to characterize the abscissa of summability for various methods.

The calculations necessary to extend Theorem 1 are of well-known kind but a good deal more complicated than those involved in Theorem 1. Since they have not been carried out to give definite results I shall not follow this line further.

The statement of Theorem 1 would be more elegant if the hypothesis that the right side of (9) is uniformly bounded could be dropped. It can certainly be replaced by other growth conditions, whose purpose is to establish a connection between the functions $G_{\sigma}$ for various values of $\sigma$. Apparently the condition may be very weak, but must bear uniformly on $\sigma$. So for example it is clear from the other hypothesis of the theorem that $f(\sigma+i t)=o(t)$ for each $\sigma>\beta$, but not that the condition holds uniformly, which would easily be enough to prove the theorem.

\section{Perron's Formula}

We write formula (3) in the equivalent form

$$
e^{-x \sigma} F(x)=\lim _{\omega \rightarrow \infty} \frac{1}{2 \pi} \int_{-\omega}^{\omega} \frac{f(\sigma+i t)}{\sigma+i t} e^{i x t} d t .
$$

Evidently (12) expresses $e^{-x \sigma} F(x)$ as the inverse transform of its Fourier transform. Now for $\sigma>\sigma_{1}$ this function is summable and has only simple discontinuities, where its value has been defined to be the average of its limits from the left and the right. So the validity of (12) is assured by a classical theorem on the inversion of Fourier integrals [6, Vol. II, p. 242].

\section{The discrete sum}

Define the periodic function

$$
H(x)=2 \pi \sum_{n=-\infty}^{\infty} e^{-(x+2 \pi n) \sigma} F(x+2 \pi n) \quad\left(\sigma>\sigma_{1}\right) .
$$


(The sum over negative indices is really finite, because $F(x)=0$ for $x<\lambda_{1}$ ). $H$ is defined at every point, has limits from left and right, and is the average of these limits. Moreover $H$ is summable and has Fourier series

$$
H(x) \sim \sum_{-\infty}^{\infty} \frac{f(\sigma+i k)}{\sigma+i k} e^{i k x} \quad\left(\sigma>\sigma_{1}\right)
$$

By analogy with Perron's Theorem we should expect $H$ to be the sum of its Fourier series. This is probably not true generally, but it can be proved under a mild hypothesis on the exponents $\lambda_{n}$ :

Theorem 2. Let $r_{k}$ denote the number of $\lambda_{j}$ satisfying

$$
2 \pi k \leqslant \lambda_{j}<2 \pi(k+1) .
$$

Denote by $\propto$ the infimum of positive numbers $\beta$ such that

$$
\sum_{k} e^{-2 \pi k \beta} \log ^{+} r_{k}<\infty .
$$

Then $H$ is the limit of the symmetric partial sums of its Fourier series provided that $\sigma>\sigma_{1}+\alpha$.

In the case of ordinary Dirichlet series, where $\lambda_{n}=\log n,(16)$ is true for each $\beta>0$, so the conclusion holds if merely $\sigma>\sigma_{1}$.

It is true at any rate that $H$ is the limit of the Fejer means of its Fourier series at every point [6, Vol. I, p. 89]. Therefore it will suffice to establish the convergence of the symmetric partial sums of the series in (14), the limit being necessarily $H(x)$.

We shall need a new estimate for the Dirichlet kernels

$$
D_{N}(x)=\sum_{k=-N}^{N} e^{i k x}=\frac{\sin \left(N+\frac{1}{2}\right) x}{\sin \frac{1}{2} x},
$$

which is given in the following lemma. The proof has been made both simpler and more convincing by Professor J.-P. Kahane.

Lemma 2. For any positive integer $r$, and any numbers

$$
\alpha_{1}=0<\alpha_{2}<\ldots<\alpha_{r+1}=2 \pi
$$

we have

$$
\sum_{j=1}^{r}\left|\int_{\alpha_{j}}^{\alpha_{j+1}} D_{N}(x) d x\right|<A+B \log r
$$

for $N=1,2, \ldots$, where $A$ and $B$ are absolute constants.

In the statement of the lemma we have trivially $r$ in place of $\log r$, because each term of the sum in (19) is at most $2 \pi$. Also $\log r$ could be replaced by $\log N$, which is the order of the left side of (19) if $D_{N}$ is replaced by its absolute value. For Theorem 2 we require a bound independent of $N$. It is surprising that even the weakest such estimate, $O(r)$ in place of the trivial $O(r)$, is enough to prove Theorem 2 for the class of ordinary Dirichlet series. 


\section{H. Helson, Convergent Dirichlet series}

The graph of $D_{N}$ in the interval $(O, 2 \pi)$ consists of $N+1$ arcs above the axis separated by $N$ ares below. As we count from the left or right side towards the center, the areas enclosed by the arcs with the axis decrease. If the points $\alpha_{j}$ $(2 \leqslant j \leqslant r)$ lie on zeros of $D_{N}$, namely the points $2 \pi k /(2 N+1)(k=1,2, \ldots, 2 N)$, then the sum on the left side of (19) can be estimated precisely. We shall show first that the lemma is true for these special systems of $\alpha_{j}$, and afterwards that other systems can give no larger value to the left side of (19).

Each summand in (19) is the algebraic sum of the areas of a certain number of adjacent regions lying alternately above and below the axis. This quantity is at most equal to the area of the largest of these regions, except possibly for a term where $\alpha_{j}<\pi<\alpha_{j+1}$, which is certainly less than twice the area of its largest region. Hence the sum in (19) is less than twice the combined area of the $r$ largest regions, or

$$
4 \int_{0}^{\pi r /(2 N+1)}\left|D_{N}(x)\right| d x .
$$

A simple calculation shows that this is less than $A+B \log r$, where $A$ and $B$ are independent of $N$.

Now suppose the $\alpha_{j}$ are required only to satisfy (18). The upper bound of the left side of (19) over all such systems, $N$ being fixed, is attained for some set of $\alpha_{j}$ which may, however, not all be distinct. We can omit redudant points, renumber the rest, and possibly reduce $r$ thereby.

Consider such an extremal system. If successive integrals

$$
\int_{\alpha_{j-1}}^{\alpha_{j}} D_{N}(x) d x, \quad \int_{\alpha_{j}}^{\alpha_{j+1}} D_{N}(x) d x
$$

have the same sign, we can replace these terms in (19) by the single one

$$
\int_{\alpha_{j-1}}^{\alpha_{j+1}} D_{N}(x) d x
$$

without any effect except to reduce further the number $r$. If any integrals vanish they can be amalgamated in the same way. We perform this operation until successive integrals (21) in (19) have alternating signs.

Then the surviving points $\alpha_{j}$ lie at zeros of $D_{N}$, aside from the end-points of the interval. Indeed, if some $\alpha_{j}$ were not on a zero, a slight change of position would increase the modulus of both integrals (21) at the same time, because they have opposite signs. This would violate the extremal property of the $\alpha_{j}$.

We have shown that the left side of (19) is less than a sum of the same type, perhaps with a smaller value of $r$, in which the $\alpha_{i}$ lie on the zeros of $D_{N}$ and the end-points of the interval; and that such a sum is less than $A+B \log r$, where $A$ and $B$ are absolute constants. So the lemma is proved.

We proceed with the proof of Theorem 2. For simplicity consider (14) first at $x=0$, and make the definitions

$$
S_{N}=\sum_{k=-N}^{N} \frac{f(\sigma+i k)}{\sigma+i k}
$$




$$
A_{n}=a_{1}+a_{2}+\ldots+a_{n}
$$

Then $F(x)=A_{n}$ for $\lambda_{n}<x<\lambda_{n+1}$. We have

$$
S_{N}=\frac{1}{2 \pi} \int_{0}^{2 \pi} H(x) D_{N}(x) d x=\int_{-\infty}^{\infty} e^{-x \sigma} F(x) D_{N}(x) d x=\sum_{n=1}^{\infty} A_{n} \int_{\lambda_{n}}^{\lambda_{n+1}} e^{-x \sigma} D_{N}(x) d x .
$$

The infinite series is convergent for each $N$, and we have to show $S_{N}$ converges as $N$ tends to infinity.

Denote the integral in the last member of (25) by $\varrho_{N}(n)$. By the second meanvalue theorem for integrals it can be written

$$
\varrho_{N}(n)=e^{-\lambda_{n} \sigma} \int_{\lambda_{n}}^{\lambda^{\prime} n} D_{N}(x) d x+e^{-\lambda_{n+1} \sigma} \int_{\lambda^{\prime} n}^{\lambda_{n+1}} D_{N}(x) d x,
$$

where $\lambda_{n}^{\prime}$ depends on $N$ but satisfies $\lambda_{n}<\lambda_{n}^{\prime}<\lambda_{n+1}$. Setting $\varepsilon_{n}=\lambda_{n+1}-\lambda_{n}$, $\varrho_{N}(n)$ takes the convenient form

$$
\varrho_{N}(n)=e^{-\lambda_{n} \sigma}\left\{\int_{\lambda_{n}}^{\lambda_{n} n} D_{N}(x) d x+e^{-\varepsilon_{n} \sigma} \int_{\lambda_{n}^{\prime}}^{\lambda_{n+1}} D_{N}(x) d x\right\} .
$$

Suppose that $\sigma>\sigma_{1}+\alpha$ (the constants in the statement of the theorem), and more precisely let $\sigma=\gamma+\beta$, where $\gamma>\sigma_{1}$ and $\beta>\alpha$. Then from (25)

$$
S_{N}=\sum_{n=1}^{\infty}\left(A_{n} e^{-\lambda_{n} \gamma}\right) e^{-\lambda_{n} \beta}\left(e^{\lambda_{n} \sigma} \varrho_{N}(n)\right)
$$

The factor $A_{n} e^{-\lambda_{n} \gamma}$ tends to zero with $1 / n$, because $F(x) e^{-x \gamma}$ tends to zero. Now the crucial step in the proof is to show that

$$
K(N)=\sum_{n=1}^{\infty} e^{-\lambda_{n} \beta} e^{\lambda_{n} \sigma}\left|\varrho_{N}(n)\right|<K
$$

for some constant $K$ independent of $N$. Suppose this has been proved. Then the proof is easily finished. For $\varrho_{N}(n)$ has a limit as $N$ tends to infinity, for each $n$, in consequence of the localization principle of Fourier series. Therefore each partial sum of (25) converges; and the remainder is uniformly as small as we please, by (29) and the fact that $A_{n} e^{-\lambda_{n} \gamma}$ tends to zero.

Removing the assumption that $x=0$ in (14) is not difficult. It is necessary to use the fact that for each fixed real number $u$, the sequence $\left\{\lambda_{n}-u\right\}$ behaves exactly like $\left\{\lambda_{n}\right\}$ with respect to the convergence of the sum in (16).

So we are left with the kernel of the proof. In (29) consider together the terms for which $2 \pi k \leqslant \lambda_{n}<2 \pi(k+1)$; evidently

$$
K(N) \leqslant \sum_{k} e^{-2 \pi k \beta} \sum\left|\varrho_{N}(n)\right| e^{\lambda_{n} \sigma},
$$

where the inner sum is taken over those $n$ such that $\lambda_{n}$ lies in the interval mentioned.

It follows from (27) that 
H. HeLson, Convergent Dirichlet series

$$
\left|\varrho_{N}(n)\right| e^{\lambda_{n} \sigma}<\left|\int_{\lambda_{n}}^{\lambda_{n}} D_{N}(x) d x\right|+\left|\int_{\lambda_{n}}^{\lambda_{n+1}} D_{N}(x) d x\right| .
$$

Now form the increasing sequence of points $\tau_{1}, \tau_{2}, \ldots$ consisting of all the points $\lambda_{n}, \lambda_{n}^{\prime}$, and if they are missing, also the points $2 \pi k$ which are larger than $\lambda_{1}$. We use (31) to increase the right side of (30), and then expand once more to

$$
K(N)<\sum_{k} e^{-2 \pi k \beta} \sum\left|\int_{\tau_{n}}^{\tau_{n+1}} D_{N}(x) d x\right|,
$$

where the inner sum extends over $n$ such that $2 \pi k \leqslant \tau_{n}<\tau_{n+1} \leqslant 2 \pi(k+1)$. Now Lemma 2 asserts that each inner sum is at most $A+B \log ^{+}\left(2 r_{k}+2\right)$, taking account of the fact that there may be as many as $2 r_{k}+2$ points $\tau_{n}$ between $2 \pi k$ and $2 \pi(k+1)$. This estimate is uniform in $N$, even though some of the points $\tau_{n}$ depend on $N$.

By hypothesis,

$$
K=\sum_{k} e^{-2 \pi k \beta}\left(A+B \log ^{+}\left(2 r_{k}+2\right)\right)
$$

is finite, and this concludes the proof of the theorem.

It may be amusing to illustrate Theorem 2 by applying it to the function

$$
\zeta_{1}(s)=\left(1-2^{1-s}\right) \zeta(s)=\sum_{n=1}^{\infty}(-1)^{n+1} n^{-s},
$$

the series on the right being convergent for $\sigma>0$. We have immediately for any real number $u$ and $\sigma>0$ that

$$
\sum_{k=-N}^{N} \frac{\zeta_{1}(\sigma+i u k)}{\sigma+i u k}
$$

converges as $N$ tends to infinity. By studying the associated function $H$ it is possible to obtain results about the growth of $\zeta_{1}(\sigma+i u k)$ in an elementary way. These results are much less precise than the corresponding known facts about $\zeta_{1}(\sigma+i t)$, but some of them seem to be new.

\section{The Theorem of Schnee}

Since the theorem was fully stated in the introduction, we proceed to the proof. Assume first that $\sigma$ is positive as well as larger than $\sigma_{c}$. For fixed $\sigma$ the series (1) converges unjformly on each finite interval $-\omega<t<\omega$. Therefore in (4) we can replace $f$ by its series to obtain

$$
J_{\omega}=\frac{1}{2 \omega} \int_{-\omega}^{\omega} f(\sigma+i t) e^{i \lambda t} d t=\sum_{n=1}^{\infty} a_{n} e^{-\lambda_{n} \sigma} \int_{-\omega}^{\omega} e^{i\left(\lambda-\lambda_{n}\right) t} \frac{d t}{2 \omega} .
$$

Let $k$ be the first integer such that $\lambda_{k}>\lambda$. It is elementary to verify that the partial sum of order $k$ on the right side of (36) has a limit, and gives the result of the theorem. Hence we may neglect those terms, and prove that the com- 
plementary sum tends to zero as $\omega$ tends to infinity. It comes to the same if we assume at the outset that $\lambda_{1}>\lambda$, and prove that $J_{\omega}$ tends to zero.

By evaluating the integral on the right side of (36) we find

$$
J_{\omega}=\sum_{n=1}^{\infty} a_{n} e^{-\lambda_{n} \sigma} \frac{\sin \omega\left(\lambda_{n}-\lambda\right)}{\omega\left(\lambda_{n}-\lambda\right)}
$$

With the function $F$ defined by (6) this can be written

$$
J_{\omega}=\int_{-\infty}^{\infty} e^{-x \sigma} \frac{\sin \omega(x-\lambda)}{\omega(x-\lambda)} d F(x),
$$

where actually the integration terminates at $\lambda_{1}$ on the lower side because $F(x)=0$ for $x<\lambda_{1}$. Now integrate by parts. The product term

$$
F(x) e^{-x \sigma} \frac{\sin \omega(x-\lambda)}{\omega(x-\lambda)}
$$

vanishes for $x<\lambda_{1}$; and $F(x) e^{-x_{a}}$ tends to zero as $x$ tends to infinity (because $\left.\sigma>\sigma_{1}\right)$, as does also the other factor. Hence this term makes no contribution to $J_{\omega}$.

We have therefore

$$
J_{\omega}=-\int_{-\infty}^{\infty} F(x) \frac{d}{d x}\left\{e^{-x \sigma} \frac{\sin \omega(x-\lambda)}{\omega(x-\lambda)}\right\} d x
$$

The derivative within the integral leads to several terms, more or less of the same type. Each one contain a factor $e^{-x \sigma}$ which combines with $F(x)$ to give a summable function. The terms, with one exception, have denominators of order $x$ or $x^{2}$, and containing the factor $\omega$ as well. These terms are trivially $O\left(\omega^{-1}\right)$ as $\omega$ tends to infinity. The exceptional term arose from differentiating $\sin \omega(x-\lambda)$, yielding $\omega$ in the numerator:

$$
-\int_{-\infty}^{\infty} F(x) e^{-x \sigma} \frac{\cos \omega(x-\lambda)}{(x-\lambda)} d x
$$

This quantity tends to zero by the Riemann-Lebesgue Lemma.

In the proof we had to assume that $\sigma>0$, and we wish to remove that hypothesis. If $\sigma_{c}<\sigma \leqslant 0$, consider $g(s)=f(s-\alpha)$, where $\sigma+\alpha>0$. Then the relation

$$
\lim _{\omega \rightarrow \infty} \frac{1}{2 \omega} \int_{-\omega}^{\omega} f(\sigma+i t) e^{i \lambda t} d t=\lim _{\omega \rightarrow \infty} \frac{1}{2 \omega} \int_{-\omega}^{\omega} g(\sigma+\alpha+i t) e^{i \lambda t} d t=0
$$

follows from what has been shown, and the theorem is proved. 


\section{H. Hetson, Convergent Dirichlet series}

\section{R E F E R E N C E S}

1. Bohr, H., and Cramér, H., Die neuere Entwicklung der analytischen Zahlentheorie. Enzyk. der math. Wiss. $I I$ 3, 722-849 (1923). Collected works of $\mathrm{H}$. Bohr, Vol. 3.

2. Carlson, F., Contributions à la théorie des séries de Dirichlet. I. Arkiv för Matematik, As. tronomi och Fysik 16, No. 18 (1922).

3. Landau, E., Handbuch der Lehre von der Verteilung der Primzahlen, Vol. 2, with appendix by Paul T. Bateman. New York, 1953.

4. Paley, R. E. A. C., and Wiener, N., Fourier transforms in the complex domain. Amer. Math. Soc. Coll. Pub. XIX, 1934.

5. Valiron, G., Théorie Générale des Séries de Dirichlet. Paris, 1926.

6. Zygmund, A., Trigonometric Series, Vol. I and Vol. II. Cambridge, 1959.

University of California, Berkeley, California. 